\title{
Vaccine-Associated Enhanced Viral Disease: Implications for Viral Vaccine Development
}

\author{
Scott B. Halstead ${ }^{1}$ (1) \\ Accepted: 2 August 2021 / Published online: 9 September 2021 \\ (c) The Author(s), under exclusive licence to Springer Nature Switzerland AG 2021
}

\begin{abstract}
Vaccine-associated enhanced disease (VAED) is a serious barrier to attaining successful virus vaccines in human and veterinary medicine. VAED occurs as two different immunopathologies, antibody-dependent enhancement (ADE) and vaccineassociated hypersensitivity (VAH). ADE contributes to the pathology of disease caused by four dengue viruses (DENV) through control of the intensity of cellular infection. Products of virus-infected cells are toxic. A partially protective yellow fever chimeric tetravalent DENV vaccine sensitized seronegative children to ADE breakthrough infections. A live-attenuated tetravalent whole virus vaccine in phase III testing appears to avoid ADE by providing durable protection against the four DENV. VAH sensitization by viral vaccines occurred historically. Children given formalin-inactivated measles or respiratory syncytial virus (RSV) vaccines experienced severe disease during breakthrough infections. Tissue responses demonstrated that VAH not ADE caused these vaccine safety problems. Subsequently, measles was successfully and safely contained by a live-attenuated virus vaccine. The difficulty in formulating a safe and effective RSV vaccine is troublesome evidence that avoiding VAH is a major research challenge. VAH-like tissue responses were observed during breakthrough homologous virus infections in monkeys given severe acute respiratory syndrome (SARS) or Middle East respiratory syndrome (MERS) vaccines.
\end{abstract}

\section{Key Points}

Human and veterinary vaccines may sensitize to enhanced breakthrough virus infections.

At least two mechanisms of sensitization, antibodydependent enhancement and vaccine-associated hypersensitivity, are identified.

Vaccine safety requires studies designed to identify and avoid virus vaccine-enhanced disease.
Scott B. Halstead

halsteads@erols.com

1 Rockville, MD, USA

\section{Introduction}

The COVID-19 pandemic has produced widespread speculation that antibody-dependent enhancement (ADE) may jeopardize the safety of SARS CoV-2 vaccines or immunotherapies $[1,2]$. While concerns about COVID-19 vaccine safety and efficacy may be justified, it is important to differentiate mechanisms of vaccine-associated enhanced disease (VAED). Viral vaccines have been shown to sensitize individuals to VAED by at least two distinct immunopathologic mechanisms. In humans, early in the 1960s, formalininactivated measles vaccines were introduced in the US and Europe. Within months, large numbers of vaccinated children developed 'atypical measles' [3]. A similar outcome, vaccine-associated enhanced respiratory disease (VAERD), was observed in infants 4-12 months of age, given formalininactivated respiratory syncytial virus (RSV) vaccines who a few months later were infected by RSV [4]. Recently, ADE was identified as the cause of breakthrough severe dengue disease in seronegative children who had been given a yellow fever chimeric tetravalent dengue vaccine $[5,6]$. It has been known for some time that ADE causes feline infectious peritonitis (FIP), a fatal Arthus-like pyogranulomatous 
coronavirus disease in vaccinated kittens and cats [7-9]. These two different vaccine sensitization scenarios are controlled by pathogenetic mechanisms as yet incompletely understood [10].

In assessing the safety of COVID-19 vaccines, it was established that animals given severe acute respiratory syndrome (SARS) and Middle East respiratory syndrome (MERS) vaccines and then challenged with wild coronaviruses developed pathological tissue responses similar to those of RSV and measles VAED [11, 12]. The similar pathologies of breakthrough RSV and measles in vaccinated humans and post-SARS and MERS vaccination of animals suggests they result from responses of increased severity to viral infection. This has been termed vaccineassociated hypersensitivity (VAH) [13]. When VAED literature searches are extended to domestic animals, a much broader etiological and pathological picture emerges. This review considers the nature, mechanisms, and the implications of VAED for human vaccine development. Causative mechanisms discussed include ADE occurring during natural infections and in vaccinated humans and animals, and VAH in vaccinated humans and animals. The larger complex of immunopathologies associated with breakthrough virus infections in vaccinated domestic animals is considered briefly.

\section{Vaccine-Associated Enhanced Disease (VAED)}

\subsection{Antibody-Dependent Enhancement (ADE)}

$\mathrm{ADE}$ is a generic phenomenon of Fc-receptor-bearing target cells in which increased intracellular replication occurs when infecting micro-organisms are presented as immune complexes in contrast to infection by naked micro-organisms $[14,15]$. This is called intrinsic ADE, to be distinguished from extrinsic ADE, which is an increased rate of attachment or internalization of organisms presented as IgG antibody complexes to $\mathrm{Fc}$ receptor-bearing cells compared with virus only $[14,15]$. Extrinsic ADE was recognized as an increase in Murray Valley encephalitis (MVE) virus plaque counts on chick embryo monolayers in the presence compared with the absence of MVE antibodies [16, 17]. Intrinsic ADE was discovered when monkeys immune to DENV 1, 3 or 4 were challenged with DENV 2 and circulated virus at significantly higher levels than during DENV 2 infections in susceptible animals [18].

\subsubsection{Dengue ADE in Humans During Natural Infections}

Human counterparts to in vitro and monkey in vivo ADE phenomena were identified when multiple types of DENVs were found to cause fatal epidemic dengue hemorrhagic fever (DHF) in the Philippines and Thailand in the 1950s $[19,20]$. Longitudinal studies in Thailand established that DHF accompanied a second heterotypic DENV infection and also a first DENV infection in infants born to DENV immune mothers [21, 22]. In humans with DHF, virus titers in blood were significantly increased early in secondary DENV infections, achieving titers that correlated with disease severity and differing from lower peak viremia titers and milder disease accompanying primary DENV infections [23]. An animal model counterpart to infant DHF was demonstrated when monkeys receiving low concentrations of human dengue immune cord blood antibodies developed enhanced DENV 2 viremias compared with viremias in dengue-naïve animals [18].

The mechanism by which antibodies control infection severity in humans was recognized when myeloid cells were identified as principal targets of in vivo DENV infections [24, 25]. Paradoxically, the ligation of monocyte/macrophage $\mathrm{Fc} \gamma$ receptors by IgG immune complexes was found to suppress innate immunity, liberate IL-10, and bias Th1 to Th2 responses. Intrinsic ADE may operate with many protozoan, bacterial, and viral organisms infecting a spectrum of vertebrate hosts $[10,14]$. ADE provides a unitary mechanism explaining DHF in individuals infected after a single prior DENV infection and also those circulating, passively acquired DENV antibodies [26]. Monocytes/macrophages process circulating subneutralized $\mathrm{IgG}$ dengue antibody complexes with consequent regulation of infection intensity [27]. The complexities, mechanisms, and outcomes of dengue $\mathrm{ADE}$ have been described in greater detail elsewhere [28].

In humans, ADE produces a life-threatening dengue shock syndrome (DSS), more accurately labeled dengue vascular permeability syndrome (DVPS), occurring in $\sim 2 \%$ of second heterotypic DENV infections [29]. DVPS is characterized by thrombocytopenia, altered hemostasis, activated complement, and elevated cytokine/chemokine and liver enzyme levels recognized shortly before or with defervescence. Damaged endothelium allows fluid and small macromolecules to escape circulation, and if fluid is not replaced, may lead to shock [30,31]. This clinical course aptly describes a new Coombs/Gell Type V immunopathology in which IgG immune complexes enhance microbial infections in FcR-bearing cells.

In 2015, Paul Young's group reported an analogy between the cellular biology of bacterial lipopolysaccharides (LPS) and that of DENV non-structural protein 1 (NS1) [32]. LPS is responsible for septic shock. Both molecules interact with toll-like receptor 4 (TLR 4) on the surface of monocytes, macrophages, and endothelial cells, inducing the release of a range of cytokines and chemokines. These same cytokines circulate in the blood of patients with DHF/DSS and often 
are mistakenly attributed to be the direct cause of vascular permeability [33, 34]. In vitro, NS1 results in the disruption of endothelial cell monolayer integrity. Crucially, the same observation was confirmed in an in vivo model [35]. The Harris laboratory showed that DENV 2 NS1 inoculated intravenously at physiologically relevant concentrations in sub-lethally DENV 2-infected C57BL/6 mice produced lethal vascular permeability. Vaccination of mice with DENV 2 NS1 protected them against endothelial leakage and death from lethal DENV 2 challenge. The authors conclude that DSS may be a viral protein toxicosis. NS1-mediated cytokine release could be inhibited by the TLR4 antagonist LPS-Rhodobacter sphaeroides, suggesting an avenue for therapeutic intervention. In dengue, ADE controls intensity of cellular infection. The products of virus-infected cells are the cause of disease.

Dengue ADE is not a simple process. Four different DENVs circulate as large numbers of antigenic and biological variants that participate in serial infections of very large numbers of genetically diverse humans. Antibodyenhanced secondary heterotypic DENV infections occur in 12 sequences. Of these, a first infection by DENV 1 or 3 followed by infection with DENV 2 generates DVPS at higher frequencies than with other sequences [29]. The clinical severity of a DENV infection may be moderately increased in individuals with a prior Japanese encephalitis (JE) or Zika virus infection [36, 37]. This implies that IgG JE or Zika antibodies complexed with DENVs send weaker inhibitory signals via the Fc receptor than do dengue antibodies complexed with DENVs. These differences in in vivo mechanisms are not understood and have not been reproduced in vitro. Antibodies to any flavivirus readily enhance DENV infections in Fc-receptor-bearing cell cultures [38]. Individuals experiencing infections with DENVs circulate a mix of antibodies, the majority of which are able to enhance DENV infections. Dengue ADE is expressed with greatest clinical severity in young children who have an innate endothelial fragility and in the elderly with pre-existing conditions [39]. Genetic factors may also control the clinical outcomes of dengue ADE. DVPS attack rates during secondary DENV infections of sub-Saharan Africans are significantly lower than rates in Caucasians or Asians [40].

\subsubsection{Dengue ADE in Vaccinated Humans: Yellow Fever Chimeric Vaccine}

Viable viral chimeras were obtained when genes of structural virus proteins of one flavivirus were introduced into a backbone of another [41]. This technology resulted in dengue virus attenuation leading to a candidate DENV 1-4 chimera of yellow fever $17 \mathrm{D}$ virus [42]. Grown in Vero cells, these constructs exhibited attenuation attributes in pre-clinical testing, resulting in commercial production by Sanofi Pasteur as Dengvaxia. A three-dose $(0,6$, and 12-month regimen) chimeric yellow fever tetravalent dengue vaccine was tested in a phase III efficacy trial involving 35,000 children in ten dengue-endemic countries [43]. Twenty-five months after a third dose, pooled efficacy rates were $60.3 \%$ for all symptomatic participants, $65.6 \%$ for those $9-16$ years of age, and $44.6 \%$ for those younger than 9 years. Vaccine protection against hospitalization was somewhat better, $72.6 \%$ for children of all ages, $80.75 \%$ for children 9 years and older, but $55.8 \%$ for those aged $<9$ years. Among the 2029 children who were 5 years or younger and received the vaccine, 20 were hospitalized for dengue compared with only two among 1005 controls, a relative risk of $4.95, p=0.03$. This led the manufacturer and WHO advisory groups to recommend Dengvaxia be given to children 9 years and older and directed to populations in national and regional settings with a dengue seroprevalence of $70 \%$ or greater $[43,44]$.

Based on these results, Sanofi re-examined the phase III Dengvaxia vaccine efficacy and safety [5]. While few children had been bled prior to vaccination, all were bled 13 months after the first dose. Using these sera, the serostatus at the time of vaccination was estimated by identifying DENV NS1 antibodies in a 10\% random sample of the phase III population. Five years after the first dose, protection by Dengvaxia against symptomatic dengue in all age groups fell to $34 \%$, a relative risk of 0.66 . Regardless of serostatus, in the complete phase III population, 80 vaccinated children were hospitalized with severe dengue, defined as thrombocytopenia and vascular permeability (standard criteria for DHF), compared with 62 severe cases in controls, a relative risk of 0.647 . Among 2- to 8-year-old children vaccinated as seronegatives, risks of breakthrough clinical dengue rose steeply. There were 137 hospitalizations among 1928 vaccinated children compared with 37 among 1006 controls (multiple imputation method), relative risk 1.87; $p \geq 0.05$. Among vaccinated 2- to 8-year-old seronegative children, 30 had severe dengue compared with five in the control group, relative risk $3.1 ; p \leq 0.05$. This comprises evidence of VAED due to ADE. Seronegative vaccinated 9- to 16-year-olds also experienced higher rates of hospitalization than controls. Of 3300 vaccinated and 1710 controls, 14.8 children were hospitalized with severe dengue versus 3.6 in the controls, relative risk 2.27, not significant. Absence of statistical power can be attributed to small numbers. When phase III data were applied to the Philippines, where 880,464 9-year-old children were vaccinated in 2016, among an estimated 132,070 seronegatives, it was projected during a 4-year period there would be 2241 hospitalizations with 480 severe dengue cases [6]. The Philippine press has reported deaths among vaccinated children. Data identifying pre-vaccination serostatus for any of these cases cannot be found in the public domain. That being said, in the 
Philippines, case fatality rates for children hospitalized with severe dengue are consistently around 5\%.

\subsubsection{Poorly Protective Vaccine, ADE Status Uncertain: Dengue 2 Chimeric Vaccine}

A second chimeric vaccine produced by Takeda Pharmaceuticals, TAK 003, given as two doses, incorporates prM and E genes of PDK-attenuated DENV 1, 3 and 4 into the genetic backbone of DENV 216681 PDK 53 and is grown in Vero cells [45]. The DENV 2 in TAK 003 is the same attenuated virus as in the earlier Mahidol/Aventis Pasteur tetravalent vaccine tested in phase II [46]. When grown in PDK, this DENV 2 was immunogenic and non-reactogenic in ten flavivirus non-immune US adult volunteers, eight of whom were viremic to a maximum of 4 days and all developed neutralizing antibodies that persisted for 2 years [47]. In January 2018, Takeda announced completion of a phase III trial in eight dengue endemic countries in South-East Asia and Latin America [48]. The cumulative efficacy for symptomatic dengue regardless of serostatus over 2 years was $72.7 \%$ (95\% CI 67.1-77.3) [49]. Efficacy declined from $80.2 \%$ in year 1 to $56.2 \%$ (95\% CI 42.3-66.8) in year 2, the largest decline to $24.5 \%$ (95\% CI -34.2 to 57.5$)$ in 4- to 5-yearold children, with efficacies of $60.6 \%$ (95\% CI 43.8-72.4) in 6- to 11-year-olds and $71.2 \%$ (95\% CI 41.0-85.9) in the 12- to 16-year age groups. Prevention of hospitalization over 2 years remained high, at $89.2 \%$ (95\% CI 82.4-93.3). Serotype vaccine efficacy, regardless of serostatus, was $69 \%$ for DENV 1, 90.8\% for DENV 2, 51.4\% for DENV 3, with insufficient DENV 4 cases to measure efficacy. Because TAK-003 efficacy varies by serotype, observed changes in serotype dominance in vaccine study sites may contribute to year-to-year efficacy differences. Somewhat alarmingly, compared with placebos, during year 2, vaccinated seronegative 4- to 5-year-olds experienced an increased rate of mild and hospitalized dengue illnesses including cases with hematocrit rises. Due to under-powering, this increase was not significant, but may be a warning.

\subsubsection{Dengue Vaccine ADE in Laboratory Animals: Formalin-Inactivated Whole Virus}

Dengue vaccine development started in 1929 when virus in blood or mosquitoes was inactivated with phenol, formalin, or bile and inoculated in human volunteers who were then challenged with live virus [50,51]. Since then, there have been comprehensive efforts to develop and test candidate purified formalin-inactivated dengue vaccines. Monovalent or tetravalent formalin-inactivated purified vaccines, prepared with or without adjuvants, were inoculated in rhesus monkeys then challenged with wild-type DENVs at varying intervals. All purified inactivated vaccines (PIVs) raised significant levels of neutralizing antibodies [52-54]. On challenge with live DENV, low levels of viremias or RNAemias were detected. Because viremia levels were considered to be lower than levels in diseased humans while antibody responses were fairly robust, vaccines were advanced to phase I in susceptibles and partial dengueimmunes [55-57]. The Walter Reed Army Institute of Research (WRAIR), GSK and the Oswaldo Cruz Foundation produced and tested a candidate tetravalent PIV formulated with aluminum hydroxide $(\mathrm{AlOH})$ or a GSK proprietary Adjuvant System. On short-interval challenge, vaccinated rhesus monkeys were protected [54]. When a larger group of animals were immunized then challenged with each of the four wild-type DENVs 8 months later, many vaccinated animals given either adjuvant had breakthrough viremias or RNAemias. Nearly all had anamnestic antibody responses and, compared with DENV-only controls, many had elevated levels of aspartate aminotransferase (AST) and distinctive IL-10, IL-18, IFN- $\gamma$, and IL-12 response patterns [58]. These results are the first demonstration of a vaccine clinical physiological DENV ADE response in an experimental animal model. Detection of ADE in the monkey model resulted in termination of the PIV development program.

\subsubsection{Avoiding Vaccine ADE: Molecularly Attenuated Dengue Viruses}

Recombinant DNA technology allowed removal of 30 nucleotides $(\Delta 30)$ from the non-translated region (NTR) of a DENV 4 genome, leading to reduced replication efficacy and a virus attenuated for susceptible humans [59]. For more than 20 years, scientists at the National Institute of Allergy and Infectious Diseases (NIAID) and the Johns Hopkins Bloomberg School of Public Health have designed and tested dengue vaccine candidates. DENV 1 and 4 were attenuated at $\Delta 30$, while a DENV $3 \Delta 30 / 31$ vaccine strain was achieved by deleting additional NTR nucleotides. A DENV 2 vaccine was constructed as a chimera with DENV $4 \Delta 30$. A crucial component of this development program was that each monovalent vaccine candidate was tested for immunogenicity and attenuation in seronegative human volunteers. Vaccines were constructed into two similar live attenuated tetravalent dengue vaccine candidates (LATV), designated TV003 and TV005 [60]. Twenty susceptible volunteers given a single dose of tetravalent vaccine TV003 were challenged 6 months later with DENV $2 \Delta 30$ (Tonga 74). They experienced no viremia, dengue rash, or anamnestic antibody response (defined as 'solid protection') [61]. Also, a single dose of TV003 solidly protected seronegative human volunteers to challenge with non-parental wild-typelike DENV $3 \Delta 30$ (Sleman 78) over short and long periods. Innovatively, a second vaccine dose was used as a test of protection, defined as the absence of viremia, rash, and no 
anamnestic antibody response. That LATV protection is likely to be of long duration is evidenced by a solid protection response to a booster dose of live attenuated vaccine 12 months after initial dose (Durbin A, personal communication, 28 December 2020).

These results are complemented by data that a single dose of LATV raises monospecific DENV 1-4 neutralizing antibodies that are conformationally similar to those raised by wild-type dengue virus human infections [62]. Moreover, T-cell responses to LATV closely resemble those raised following infections with wild-type dengue viruses [63]. Finally, the NIAID LATV contains genes for three of the four DENV NS1 proteins. TV003 was licensed independently to Butantan, a vaccine manufacturer in Sao Paulo, Brazil and to Merck \& Co. Inc. At Butantan, a vaccine is in the fourth year of phase III clinical testing. The rate of accruing vaccine efficacy data in Brazil was slowed by the $80 \%$ reduction in dengue cases in 2018 that followed the Zika virus epidemic of 2016-17 [64]. Release of the Brazilian phase III results are expected in 2022. Merck's plans for the development and release of the vaccine have not been announced.

\subsection{Vaccine-Associated Hypersensitivity (VAH)}

\subsubsection{VAH in RSV and Measles-Vaccinated Humans}

Susceptible children given measles or RSV vaccines subsequently exposed to wild-type viruses developed a severe viral disease characterized by eosinophilia, complement fixation, and Arthus-like pathology [3, 4, 65, 66]. The mechanism has been attributed to delayed type hypersensitivity and/or an Arthus reaction [67-69]. Lungs from fatal RSV cases in humans had high titers of virus, damage to parenchymal tissue, bronchopneumonia with atelectasis, and pneumothorax, a pulmonary neutrophilia with abundant macrophages and lymphocytes and excess eosinophils. Based on laboratory animal models, it is thought that formalin de-conformed viral antigens raised non-protective antibodies that led to a Th2 polarization of the immune response and a deficit of cytotoxic T cells. The role that eosinophils play in pathogenesis is not well understood [70]. There is no formal proof that VAERD in humans is antibody mediated [71]. Indeed, it is ironic that a human monoclonal RSV antibody given to young infants reliably prevents naturally occurring severe RSV disease. The mechanism(s) of the post-measles vaccine disease enhancement in humans and its similarity to VAERD are not established. In both, the clinical course illustrates a type III immunopathology in which foreign antigen-antibody complexes are trapped in the basement membranes of endothelial linings. Examples include acute serum sickness and glomerular nephritis [72].

\subsubsection{Laboratory Animal Model of VAH}

Initially, a lead hypothesis was that RSV vaccine pathology was an example of ADE. In a bonnet monkey model, RSV antibody increased virus infection of pulmonary macrophages [73, 74]. However, in humans in vivo, pulmonary epithelial cells, not macrophages, are prime targets of infection. An alternate model is that antibodies raised by formalin-treated viral antigens increase RSV disease severity by forming infectious immune complexes that activate complement $[67,68]$. This pathologic response affects lung function, but may also control Th2 differentiation [75]. It was thought that formaldehyde-inactivated proteins raised antibodies of reduced protective capacity because of increased reactive carbonyl groups that favored Th2 immune responses following phagocytosis by macrophages via scavenger receptors [76]. However, inactivation of RSV by methods other than formalin also sensitized experimental animals to enhanced disease. Mice immunized with RSV treated with ultraviolet (UV) light, purified fusion (F) protein, or a vaccinia RSV replicative construct experienced enhanced disease following challenge with wild-type virus $[72,77]$. It was found that toll receptor (TLR) activation was required for avidity maturation [72]. When MyD88, a downstream adaptor of most TLRs, including TLR 4 and 7, was present, inoculation of WT RSV resulted in avid and protective antibody responses. In MyD88 ${ }^{-{ }^{-}}$mice, inoculation with this immunogen was not protective. Passive cellular transfer experiments using MyD88 $8^{-/-}$mice revealed that TLR stimulation occurred in B lymphocytes. Of interest, when UV-inactivated virus was administered along with the TLR agonists lipopolysaccharide (TLR 4 agonist) and poly (I:C) + poly U (TLR 3, 7 agonists) as opposed to use of alum (a TLR-independent adjuvant), mice were protected against RSV challenge.

Both high and low avidity antibodies were directed to the same amino acid epitope of F protein (aa 422-438). In mice, formalin-deconformed RSV epitopes resulted in a failure of avidity maturation, evidenced by sub-optimal maturation of dendritic cells and reduced production of activation factors, CD 40, CD 80, and CD 86, and decreased germinal center formation in lymph nodes [72]. Low avidity antibody responses to UV-treated F protein were converted to high avidity when antigen was administered on 5 consecutive days.

A monkey model of RSV vaccine immunopathology provided important insights into disease-producing mechanisms. As in mice, formalin inactivation of RSV yielded an antigen that poorly activated B-cell TLRs, exhibited defective affinity maturation, and produced non-protective antibodies $[72,78]$. In addition, there was a dissociation of neutralizing and ELISA antibody responses, enhanced interleukin IL-4, IL-5, and IL-13 responses, and tissue 
eosinophilia [79-81]. It was suspected that T cells might play a role in RSV lung pathology following administration of killed vaccine when it was established that specific antibodies to formalin-inactivated RSV, in the absence of CD4+ and CD8+ T cells, were not sufficient to cause disease enhancement [82]. In addition, T-cell responses to RSV contribute to tissue pathology and are identifiable once cellular infection is well established. T cells signal the host's attempts to contain and eliminate virus-infected cells. Of relevance, cynomolgus monkeys given a formalin-inactivated vaccine then challenged with wild-type human metapneumovirus, a member of the RSV family, developed clinical and pathological responses suggestive of immune-mediated disease [81].

\subsubsection{VAH in SARS CoV-Vaccinated Animals}

VAH has been documented in a number of SARS-CoV-1 animal models. In a mouse model, pre-clinical testing of SARS-CoV-1 vaccines led to pulmonary immunopathology upon challenge with SARS-CoV-1 [83, 84]. Vaccines sensitizing to an immunopathologic response to live virus challenge include inactivated whole viruses, inactivated viruses with adjuvant, and a recombinant DNA spike (S) protein vaccine in a virus-like particle (VLP) vaccine. Severe pneumonia was observed in mice vaccinated with nucleocapsid protein after challenge with SARS-CoV-1 [85]. Enhanced hepatitis was described in a ferret model using a vaccine constructed with a recombinant modified vaccinia virus Ankara (rMVA) expressing the SARS CoV-1 spike protein [86]. Dominant SARS CoV-1 epitopes produced enhanced tissue responses in vaccinated rhesus macaques challenged with wild virus [87]. VAH may be classified as a Coombs type III antigen hypersensitivity. A similar pathological response has been observed in several species of laboratory animals immunized with various other SARS and MERS $\mathrm{CoV}$ constructs, with and without adjuvants, then challenged with live virus $[11,83]$.

\subsubsection{Avoiding VAED}

Inactivated measles VAED was successfully avoided with the introduction of a durable single-dose live-attenuated measles virus. While live measles vaccine does not prevent respiratory tract super-infection, it effectively prevents measles disease accompanying reinfection [88]. Evidence of broad and sustained protection of humans by a liveattenuated DENV vaccine is not yet available, but successful DENV 2 and 3 challenge studies of seronegative volunteers given a single dose of the NIAID tetravalent live-attenuated DENV vaccine suggests the NIAID live-attenuated DENVs will protect and will not sensitize to dengue ADE.
For many reasons, attempts to introduce live-attenuated RSV vaccines have not been successful. One reason has been the difficulty of achieving robust immune responses in young infants who circulate maternal RSV antibodies. Based on studies in animal models, RSV intravenous immune globulin was introduced and subsequently replaced by humanized monoclonal antibodies directed at antigenic site II on the RSV F protein. These antibodies, given to premature infants and infants with developmental conditions, have been effective in reducing severe and fatal disease and are used in standard practice. Importantly, consistent with VAH, not $\mathrm{ADE}$, as the pathogenic mechanism, the waning of passively acquired RSV antibodies does not sensitize infants to severe disease [7-9].

\section{Discussion}

There is a large literature of vaccine failures in veterinary medicine, some of which are accompanied by enhanced breakthrough disease [89-104]. These pose a continuing challenge to attaining safe and effective vaccines. In veterinary medicine, viral vaccine sensitization is due both to $\mathrm{ADE}$ and $\mathrm{VAH}$, with many iterations and mechanisms as yet unsolved. Table 1 is a partial list of veterinary viral vaccines exhibiting in vitro ADE or VAH-like tissue responses. It should be noted that, regardless of VAED response, viruses of many genera exhibit in vitro ADE. This test cannot be used to distinguish vaccine immunopathologies. Several examples of VAED are illustrative.

Feline coronaviruses exist in two forms. Feline enteric coronavirus is a pathogen of minor significance. A spontaneous mutation of this virus results in a feline infectious peritonitis virus (FIPV) that replicates in peritoneal macrophages, producing peritonitis and occasionally FIP, a fatal Arthuslike pyogranulomatous disease in kittens and cats. ADE has long been identified as the disease-enhancing mechanism [7-9]. That antibodies are pathogenic is evidenced by observations that kittens who have acquired passive maternal FIPV antibodies develop a more rapid and fulminant disease following challenge with FIPV than do seronegatives [8]. Disease enhancement has been demonstrated in cats that were infected in the presence of vaccine-derived humoral immunity directed against the spike protein of FIPV [89]. Similarly, cats immunized with a recombinant vaccinia virus expressing the spike protein of FIPV died earlier than control animals [89]. In adult cats, FIPV develops during chronic infections with feline coronaviruses after FECV mutates to FIPV, gaining macrophage tropism [90]. As antibody responses are mounted to FIPV infection, disease severity is enhanced [9]. In summary, antibody responses to FIPV during the course of infection and the passive transfer of antibodies from natural infections and immunization 
Table 1 Observed in vitro ADE and enhanced viral diseases in animals sensitized by administration of vaccines

\begin{tabular}{|c|c|c|c|c|}
\hline & Viruses/host & In vitro $A D E$ & In vivo $\mathrm{VAED}$ & References \\
\hline \multicolumn{5}{|l|}{ RNA Virus Group } \\
\hline \multirow[t]{2}{*}{ Orthomyxoviridae } & Influenza A, mice & + & VAH (mouse model) & {$[105]$} \\
\hline & Influenza A, pigs & - & VAH & [103] \\
\hline \multirow[t]{2}{*}{ Paramyxoviridae } & RSV, human & + & VAH & {$[3,106]$} \\
\hline & Measles, human & + & VAH & \\
\hline Rhabdoviridae & Rabies, human & + & Accelerated disease onset & {$[107,108]$} \\
\hline Coronaviridae & Feline infectious peritonitis & + & $\mathrm{ADE}$ & [8] \\
\hline \multirow[t]{2}{*}{ Arteriviridae } & PRRSV & + & ADE & {$[98]$} \\
\hline & Simian HF & + & VAH? & [109] \\
\hline \multirow[t]{3}{*}{ Retroviridae } & HIV & + & ADE? & {$[110-112]$} \\
\hline & Equine infectious anemia & + & VAH & \\
\hline & Caprine arthritis & + & VAH & \\
\hline \multicolumn{5}{|l|}{ DNA Virus Group } \\
\hline Parvoviridae & Aleutian disease of mink & + & $\mathrm{ADE}$ & [94] \\
\hline
\end{tabular}

$A D E$ antibody-dependent enhancement, $H I V$ human immunodeficiency virus, PRRSV porcine reproductive and respiratory system virus, $R S V$ respiratory syncytial virus, $V A E D$ vaccine-associated enhanced disease, $V A H$ vaccine-associated hypersensitivity

with killed or recombinant vaccines all have led to enhanced FIPV disease. Enhanced disease severity has been attributed to the generation of non-neutralizing antibodies.

Aleutian disease of mink (AMD) is caused by a pathogenic parvovirus that replicates in macrophages and circulates in the blood, principally as fully infectious immune complexes [94]. AMD virus causes a persistent infection associated with immune complex disease, hypergammaglobulinemia, and high levels of antiviral antibodies. A fatal glomerulonephritis results from the deposition of these soluble immune complexes on renal glomerular membranes or walls of capillary blood vessels. ADE has been suggested to be an important contributing factor to the pathogenesis of AMD [91]. Despite the presence of antibodies, the virus is not cleared in vivo. Pre-existing antibodies may enhance viral infections by Fc-receptor-mediated ADE but the mechanism that underlies AMD has not been fully defined. Three models have been proposed, including (i) interactions between antibody and FcR, complement $\mathrm{C} 3$ fragment and $\mathrm{CR}$, or between $\mathrm{C} 1 \mathrm{q}$ and $\mathrm{C} 1 \mathrm{qR}$, which promotes viral attachment to cells; (ii) suppression of IFN- $\gamma$-mediated host-cell antiviral gene expression by the up-regulation of negative regulators of pathogen pattern recognition; and (iii) the promotion of early IL-10 secretion. There may be a role of cytokine IL-6 in ADE-mediated disease [92]. Virus replicates in macrophages and large amounts of non-neutralizing antibodies are produced in infected mink [93]. These, in turn, produce infectious immune complexes, which lead to increased infection of macrophages. In related work, passive transfer of virus-specific antibody at the peak of viral replication resulted in foci of necrosis around virus-infected cells, a reaction thought to be due to complement-mediated cytolysis [94]. Infectious immune complexes resulted in enhanced infection of mink peritoneal macrophages [95]. Inoculation of an experimental killed AMDV vaccine failed to produce detectable neutralizing antibodies, but eight of ten of these vaccinated mink — but no control animals—developed AMD on challenge with live virus [94].

Porcine Reproductive and Respiratory Syndrome (PRRS) is caused by members of Arterivirus, a positive strand RNA virus genus that includes equine arteritis and simian hemorrhagic fever viruses [96]. PRRS virus (PRRSV) was recognized in East Germany in 1980 with PRRSV-1 virus isolated in the Netherlands in 1991. Another virus, PRRSV-2, was recognized in the USA in the mid-1980s and called 'mystery swine disease.' It has also been called blue ear disease. The two prototype strains of PRRSV, European and North American, cause similar clinical symptoms, but are distinct viral genotypes whose genomes diverge by approximately $40 \%$. The genetic variation among the viruses varies geographically, increasing the difficulty of vaccine development. For reasons not understood, the clinical picture varies from one herd to another. For every three herds that are exposed to PRRSV for the first time, one will show no recognizable disease, the second may show mild disease, and the third moderate to severe disease. The better the health status of the herd, the less severe the disease observed. There is evidence that PRRSV mutates as it multiplies to evolve strains that are highly virulent and others that are not. PRRSV has a particular affinity for the macrophages in the lung [97]. The virus multiplies in then disables and kills macrophages. ADE in vivo has been demonstrated by challenging animals 
sensitized by passively transferred antibodies [98, 99]. Reminiscent of dengue, the literature on pathogenesis focuses heavily on the disease-producing role of cytokines [97]. PRRSV infects lymphoid tissues and is often chronic. Infected animals are at increased risk for a variety of microbial infections. Once a herd is infected the virus tends to remain present and active indefinitely. Some live-attenuated vaccines have provided short-term protection. Enhanced viremia and tissue infection has been described in vaccinated animals experiencing breakthrough PRRSV infections [100]. Investigators have attributed vaccine failure to ADE [101].

Influenza A viruses cause acute respiratory infections, resulting in severe economic losses to domestic animals. Although influenza pandemics in birds create headlines, there are no reports of vaccine-enhanced disease [102]. However, VAED is a well-established outcome of influenza vaccines of swine. Swine influenza results in significant economic losses for global pig production. Three subtypes of influenza A viruses of swine (IAV-S) co-circulate worldwide: H1N1, H3N2, and H1N2. The origin, genetic background, and antigenic properties of IAV-S vary considerably from region to region. Pigs have played a role in the adaptation of avian influenza A viruses from avian reservoirs to humans and other mammalian hosts. Pigs may be a 'mixing vessel' in which influenza viruses from various origins may reassert to generate novel progeny viruses capable of replicating and spreading among humans. For these many reasons, efforts are made to control porcine influenza by vaccines. It is well established that inactivated whole virion vaccines sensitize pigs to a breakthrough respiratory syndrome similar to VAH [103].

The challenge of achieving vaccines that safely and durably protect against human RSV and influenza is reminiscent of the difficulty in obtaining vaccines for diverse viral diseases that threaten commercial agriculture. Many of those who have been recently introduced to the dengue literature mistakenly assume that antibody-enhanced infection is itself a pathology. It is not. ADE controls the rate of intracellular virus production and total number of cells infected. It is NS1, a circulating viral toxin of endothelial cells, that produces the dengue shock syndrome, an analog of bacterial endotoxin septic shock. The mechanism(s) by which viral immune complexes, once introduced into FcR-bearing cells, affect replicative outcome are not well understood and have attracted surprisingly little interest [104]. Inflammatory responses following the introduction of pathogenic microorganisms into partially immune hosts are difficult to unravel as illustrated in RSV VAH animal models. Future research should approach ADE and VAH as independent systems, one involving infection amplification and the other leading to a pathologic process.

\section{Declarations}

Funding no funding was received.

Conflict of interest Scott Halstead has no conflicts of interest to declare.

Ethics approval Not applicable.

Consent to participate/publish Not applicable.

Availability of data and material Not applicable.

Code availability Not applicable.

Author contributions Scott Halstead is the sole author.

\section{References}

1. Arvin AM, Fink K, Schmid MA, Cathcart A, Spreafico $\mathrm{R}$, Havenar-Daughton $\mathrm{C}$, et al. A perspective on potential antibody-dependent enhancement of SARS-CoV-2. Nature. 2020;584(7821):353-63.

2. Ricke DO. Two different antibody-dependent enhancement (ADE) risks for SARS-CoV-2 antibodies. Front Immunol. 2021;12:640093.

3. Fulginiti VA, Eller JJ, Downie AW, Kempe CH. Altered reactivity to measles virus. Atypical measles in children previously immunized with inactivated measles virus vaccines. JAMA. 1967;202(12):1075-80.

4. Chin J, Magoffin RL, Shearer LA, Schieble JH, Lennette EH. Field evaluation of a respiratory syncytial virus vaccine and a trivalent parainfluenza virus vaccine in a pediatric population. Am J Epidemiol. 1969;89:449-63.

5. Sridhar S, Luedtke A, Langevin E, Zhu M, Bonaparte M, Machabert $\mathrm{T}$, et al. Effect of dengue serostatus on dengue vaccine safety and efficacy. N Engl J Med. 2018;379(4):327-40.

6. Halstead SB, Russell PK, Katzelnick LC, Markoff L, Aguiar M, Dans LR, et al. Ethics of a partially effective dengue vaccine: lessons from the Philippines. Vaccine. 2020;38(35):5572-6.

7. Pedersen NC, Boyle JF. Immunologic phenomena in the effusive form of feline infectious peritonitis. Am J Vet Res. 1980;41:868-76.

8. Weiss RC, Scott FW. Antibody-mediated enhancement of disease in feline infectious peritonitis: comparisons with dengue hemorrhagic fever. Comp Immune Microbiol Infect Dis. 1981;4:175-88.

9. Pedersen NC. An update on feline infectious peritonitis: diagnostics and therapeutics. Vet J. 2014;201(2):133-41.

10. Ubol S, Halstead SB. How innate immune mechanisms contribute to antibody-enhanced viral infections. Clin Vaccine Immunol. 2010;17(12):1829-35.

11. Bolles M, Deming D, Long K, Agnihothram S, Whitmore A, Ferris $\mathrm{M}$, et al. A double-inactivated severe acute respiratory syndrome coronavirus vaccine provides incomplete protection in mice and induces increased eosinophilic proinflammatory pulmonary response upon challenge. J Virol. 2011;85(23):12201-15.

12. Liu L, Wei Q, Lin Q, Fang J, Wang H, Kwok H, et al. Anti-spike IgG causes severe acute lung injury by skewing macrophage responses during acute SARS-CoV infection. JCI Insight. 2019;4(4):e123158. 
13. Halstead SB, Katzelnick L. COVID-19 vaccines: should we fear ADE? J Infect Dis. 2020;222(12):1946-50.

14. Halstead SB, Mahalingam S, Marovich MA, Ubol S, Mosser DM. Intrinsic antibody-dependent enhancement of microbial infection in macrophages: disease regulation by immune complexes. Lancet Infect Dis. 2010;10(10):712-22.

15. Boonnak K, Dambach KM, Donofrio GC, Marovich MA. Cell type specificity and host genetic polymorphisms influence antibody dependent enhancement of dengue virus infection. J Virol. 2011;85(4):1671-83.

16. Hawkes RA. Enhancement of the infectivity of arboviruses by specific antisera produced in domestic fowls. Aust J Exp Biol Med Sci. 1964;42:465-82.

17. Kliks S, Halstead SB. An explanation for enhanced virus plaque formation in chick embryo cells. Nature. 1980;285:504-5.

18. Halstead SB. In vivo enhancement of dengue virus infection in Rhesus monkeys by passively transferred antibody. J Infect Dis. 1979;140(4):527-33.

19. Quintos FN, Lim LE, Juliano L, Reyes A, Lacson P. Hemorrhagic fever observed among children in the Philippines. Philipp J Pediatr. 1954;3(3):1-19.

20. Hammon WM, Rudnick A, Sather GE, Rogers KD, Morse LJ. New hemorrhagic fevers of children in the Philippines and Thailand. Trans Assoc Am Phys. 1960;73:140-55.

21. Halstead SB, Nimmannitya S, Yamarat C, Russell PK. Hemorrhagic fever in Thailand; recent knowledge regarding etiology. Jpn J Med Sci Biol. 1967;20:96-103.

22. Halstead SB, Nimmannitya S, Cohen SN. Observations related to pathogenesis of dengue hemorrhagic fever. IV. Relation of disease severity to antibody response and virus recovered. Yale J Biol Med. 1970;42:311-28.

23. Vaughn DW, Green S, Kalayanarooj S, Innis BL, Nimmannitya $\mathrm{S}$, Suntayakorn S, et al. Dengue viremia titer, antibody response pattern, and virus serotype correlate with disease severity. J Infect Dis. 2000;181(1):2-9.

24. Halstead SB, O'Rourke EJ. Dengue viruses and mononuclear phagocytes. I. Infection enhancement by non-neutralizing antibody. J Exp Med. 1977;146:201-17.

25. Aye KS, Charngkaew K, Win N, Wai KZ, Moe K, Punyadee N, et al. Pathologic highlights of dengue hemorrhagic fever in 13 autopsy cases from Myanmar. Hum Pathol. 2014;45(6):1221-33.

26. Halstead SB. Pathogenesis of dengue: challenges to molecular biology. Science. 1988;239:476-81.

27. Halstead SB, O'Rourke EJ, Allison AC. Dengue viruses and mononuclear phagocytes. II. identity of blood and tissue leukocytes supporting in vitro infection. J Exp Med. 1977;146:218-28.

28. Halstead SB. Dengue antibody-dependent enhancement: knowns and unknowns. Microbiol Spectrosc. 2014;2(6). https://doi.org/ 10.1128/microbiolspec.AID-0022-2014.

29. Guzman MG, Alvarez M, Halstead SB. Secondary infection as a risk factor for dengue hemorrhagic fever/dengue shock syndrome: an historical perspective and role of antibody-dependent enhancement of infection. Arch Virol. 2013;158(7):1445-59.

30. Cohen SN, Halstead SB. Shock associated with dengue infection. I. Clinical and physiologic manifestations of dengue hemorrhagic fever in Thailand, 1964. J Pediatr. 1966;68:448-56.

31. Bokisch VA, Top FH Jr, Russell PK, Dixon FJ, Muller-Eberhard HJ. The potential pathogenic role of complement in dengue hemorrhagic shock syndrome. N Engl J Med. 1973;289:996-1000.

32. Modhiran N, Watterson D, Muller DA, Panetta AK, Sester DP, Liu L, et al. Dengue virus NS1 protein activates cells via Tolllike receptor 4 and disrupts endothelial cell monolayer integrity. Sci Transl Med. 2015;7(304):304ra142.

33. Chaturvedi UC, Agarwal R, Elbishbishi EA, Mustafa AS. Cytokine cascade in dengue hemorrhagic fever: implications for pathogenesis. FEMS Immunol Med Microbiol. 2000;28(3):183-8.

34. Rothman A. Dengue immune response: low affinity, high febrility. Nat Med. 2003;7:820-2.

35. Beatty PR, Puerta-Guardo H, Killingbeck SS, Glasner DR, Hopkins K, Harris E. Dengue virus NS1 triggers endothelial permeability and vascular leak that is prevented by NS1 vaccination. Sci Transl Med. 2015;7(304):304ra141.

36. Anderson KB, Gibbons RV, Thomas SJ, Rothman AL, Nisalak A, Berkelman RL, et al. Preexisting Japanese encephalitis virus neutralizing antibodies and increased symptomatic dengue illness in a school-based cohort in Thailand. PLoS Negl Trop Dis. 2011;5(10):e1311.

37. Katzelnick LC, Narvaez C, Arguello S, Lopez Mercado B, Collado $\mathrm{D}$, Ampie $\mathrm{O}$, et al. Zika virus infection enhances future risk of severe dengue disease. Science. 2020;369(6507):1123-8.

38. Halstead SB, Porterfield JS, O'Rourke EJ. Enhancement of dengue virus infection in monocytes by flavivirus antisera. Am J Trop Med Hyg. 1980;29(4):638-42.

39. WHO. Dengue: guidelines for diagnosis, treatment prevention and control. WHO; 2009.

40. Sierra B, Triska P, Soares P, Garcia G, Perez AB, Aguirre E, et al. OSBPL10, RXRA and lipid metabolism confer African-ancestry protection against dengue haemorrhagic fever in admixed Cubans. PLoS Pathog. 2017;13(2):e1006220.

41. Bray M, Men R, Lai CJ. Monkeys immunized with intertypic chimeric dengue viruses are protected against wild-type virus challenge. J Virol. 1996;70:4162-6.

42. Guirakhoo F, Arroyo J, Pugachev KV, Miller C, Zhang ZX, Weltzin R, et al. Construction, safety, and immunogenicity in nonhuman primates of a chimeric yellow fever-dengue virus tetravalent vaccine. J Virol. 2001;75(16):7290-304.

43. Hadinegoro SR, Arredondo-Garcia JL, Capeding MR, Deseda C, Chotpitayasunondh T, Dietze R, et al. Efficacy and long-term safety of a dengue vaccine in regions of endemic disease. $\mathrm{N}$ Engl J Med. 2015;373(13):1195-206.

44. Who. Global advisory committee on vaccine safety, 15-16 June 2016. Wkly Epidemiol Rec. 2016;91(28/29):341-8.

45. Huang CY, Kinney RM, Livengood JA, Bolling B, Arguello JJ, Luy BE, et al. Genetic and phenotypic characterization of manufacturing seeds for a tetravalent dengue vaccine (DENVax). PLoS Negl Trop Dis. 2013;7(5):e2243.

46. Yoksan S. Short history of dengue and Mahidol dengue vaccine. South East Asian J Trop Med Pub Health. 2017;48(Supplement 1):20-32.

47. Vaughn DW, Hoke CH Jr, Yoksan S, LaChance R, Innis BL, Rice RM, et al. Testing of a dengue 2 live-attenuated vaccine (strain 16681 PDK 53) in ten American volunteers. Vaccine. 1996;14(4):329-36.

48. Biswal S, Reynales H, Saez-Llorens X, Lopez P, Borja-Tabora C, Kosalaraksa P, et al. Efficacy of a tetravalent dengue vaccine in healthy children and adolescents. N Engl J Med. 2019;381(21):2009-19.

49. López-Medina E, Biswal S, Saez-Llorens X, Borja-Tabora C, Bravo L, Sirivichayakul C, et al. Efficacy of a dengue vaccine candidate (TAK-003) in healthy children and adolescents two years after vaccination. J Infect Dis. 2020:jiaa761. https://doi. org/10.1093/infdis/jiaa761.

50. Simmons JS, St John JH, Reynolds FHK. Experimental studies of dengue. Philipp J Sci. 1931;44:1-252.

51. Blanc G, Caminopetros J. Recherches experimentales sur la dengue. Ann Inst Pasteur (Paris). 1930;44(4):367-436.

52. Putnak R, Cassidy K, Conforti N, Lee R, Sollazzo D, Truong $\mathrm{T}$, et al. Immunogenic and protective response in mice immunized with a purified, inactivated, Dengue- 2 virus vaccine 
prototype made in fetal rhesus lung cells. Am J Trop Med Hyg. 1996;55(5):504-10.

53. Putnak RJ, Coller BA, Voss G, Vaughn DW, Clements D, Peters I, et al. An evaluation of dengue type-2 inactivated, recombinant subunit, and live-attenuated vaccine candidates in the rhesus macaque model. Vaccine. 2005;23(35):4442-52.

54. Fernandez S, Thomas SJ, De La Barrera R, Im-Erbsin R, Jarman RG, Baras B, et al. An adjuvanted, tetravalent dengue virus purified inactivated vaccine candidate induces long-lasting and protective antibody responses against dengue challenge in rhesus macaques. Am J Trop Med Hyg. 2015;92(4):698-708.

55. Martinez LJ, Lin L, Blaylock JM, Lyons AG, Bauer KM, De La Barrera R, et al. Safety and immunogenicity of a dengue virus serotype-1 purified-inactivated vaccine: results of a phase 1 clinical trial. Am J Trop Med Hyg. 2015;93(3):454-60.

56. Diaz C, Lin L, Martinez LJ, Eckels KH, Campos M, Jarman RG, et al. Phase 1 randomized study of a tetravalent dengue purified inactivated vaccine in healthy adults from Puerto Rico. Am J Trop Med Hyg. 2018;98(5):1435-43.

57. Lin L, Lyke KE, Koren M, Jarman RG, Eckels KH, Lepine E, et al. Safety and immunogenicity of an AS03(B)-adjuvanted inactivated tetravalent dengue virus vaccine administered on varying schedules to healthy U.S. adults: a phase $1 / 2$ randomized study. Am J Trop Med Hyg. 2020;103(1):132-41.

58. Borges MB, Marchevsky RS, Carvalho Pereira R, da Silva Mendes Y, Almeida Mendes LG, Diniz-Mendes L, et al. Detection of post-vaccination enhanced dengue virus infection in macaques: an improved model for early assessment of dengue vaccines. PLoS Pathog. 2019;15(4):e1007721.

59. Durbin AP, Karron RA, Sun W, Vaughn DW, Reynolds MJ, Perreault JR, et al. Attenuation and immunogenicity in humans of a live dengue virus type- 4 vaccine candidate with a 30 nucleotide deletion in its 3'-untranslated region. Am J Trop Med Hyg. 2001;65:405-13.

60. Kirkpatrick BD, Durbin AP, Pierce KK, Carmolli MP, Tibery CM, Grier PL, et al. Robust and balanced immune responses to all 4 dengue virus serotypes following administration of a single dose of a live attenuated tetravalent dengue vaccine to healthy, flavivirus-naive adults. J Infect Dis. 2015;212(5):702-10.

61. Kirkpatrick BD, Whitehead SS, Pierce KK, Tibery CM, Grier PL, Hynes NA, et al. The live attenuated dengue vaccine TV003 elicits complete protection against dengue in a human challenge model. Sci Transl Med. 2016;8(330):330ra36.

62. Tsai WY, Durbin A, Tsai JJ, Hsieh SC, Whitehead S, Wang WK. Complexity of neutralization antibodies against multiple dengue viral serotypes after heterotypic immunization and secondary infection revealed by in-depth analysis of cross-reactive antibodies. J Virol. 2015;89(14):7348-62.

63. Angelo MA, Grifoni A, O'Rourke PH, Sidney J, Paul S, Peters $\mathrm{B}$, et al. Human $\mathrm{CD} 4+\mathrm{T}$ cell responses to an attenuated tetravalent dengue vaccine parallel those induced by natural infection in magnitude, HLA restriction, and antigen specificity. J Virol. 2017;91(5).

64. Lopes TRR, Silva CS, Pastor AF, Silva Junior JVJ. Dengue in Brazil in 2017: what happened? Rev Inst Med Trop Sao Paulo. 2018;60:e43.

65. Buser F. Side reaction to measles vaccination suggesting the arthus phenomenon. N Engl J Med. 1967;277:250-1.

66. Kim HW, Canchola JG, Brandt CD, Pyles G, Chanock K, Jensen $\mathrm{K}$, et al. Respiratory syncytial virus disease in infants despite prior administration of antigenic inactivated vaccine. Am J Epidemiol. 1969;89:422-34.

67. Polack FP, Auwaerter PG, Lee SH, Nousari HC, Valsamakis A, Leiferman KM, et al. Production of atypical measles in rhesus macaques: evidence for disease mediated by immune complex formation and eosinophils in the presence of fusion-inhibiting antibody. Nat Med. 1999;5(6):629-34.

68. Polack FP, Teng MN, Collins PL, Prince GA, Exner M, Regele $\mathrm{H}$, et al. A role for immune complexes in enhanced respiratory syncytial virus disease. J Exp Med. 2002;196(6):859-65.

69. Polack FP. Atypical measles and enhanced respiratory syncytial virus disease (ERD) made simple. Pediatr Res. 2007;62(1):111-5.

70. Acosta PL, Caballero MT, Polack FP. Brief history and characterization of enhanced respiratory syncytial virus disease. Clin Vaccine Immunol. 2015;23(3):189-95.

71. Polack FP, Lydy SL, Lee SH, Rota PA, Bellini WJ, Adams RJ, et al. Poor immune responses of newborn rhesus macaques to measles virus DNA vaccines expressing the hemagglutinin and fusion glycoproteins. Clin Vaccine Immunol. 2013;20(2):205-10.

72. Delgado MF, Coviello S, Monsalvo AC, Melendi GA, Hernandez JZ, Batalle JP, et al. Lack of antibody affinity maturation due to poor Toll-like receptor stimulation leads to enhanced respiratory syncytial virus disease. Nat Med. 2009;15(1):34-41.

73. Ponnuraj EM, Springer J, Hayward AR, Wilson H, Simoes EA. Antibody-dependent enhancement, a possible mechanism in augmented pulmonary disease of respiratory syncytial virus in the Bonnet monkey model. J Infect Dis. 2003;187(8):1257-63.

74. Gimenez HB, Chisholm S, Dornan J, Cash P. Neutralizing and enhancing activities of human respiratory syncytial virus-specific antibodies. Clin Diagn Lab Immunol. 1996;3(3):280-6.

75. Anderson CF, Mosser DM. Cutting edge: biasing immune responses by directing antigen to macrophage Fc gamma receptors. J Immunol. 2002;168(8):3697-701.

76. Moghaddam A, Olszewska W, Wang B, Tregoning JS, Helson R, Sattentau QJ, et al. A potential molecular mechanism for hypersensitivity caused by formalin-inactivated vaccines. Nat Med. 2006;12(8):905-7.

77. Olszewska W, Suezer Y, Sutter G, Openshaw PJ. Protective and disease-enhancing immune responses induced by recombinant modified vaccinia Ankara (MVA) expressing respiratory syncytial virus proteins. Vaccine. 2004;23(2):215-21.

78. Johnson TR, Rao S, Seder RA, Chen M, Graham BS. TLR9 agonist, but not TLR7/8, functions as an adjuvant to diminish FI-RSV vaccine-enhanced disease, while either agonist used as therapy during primary RSV infection increases disease severity. Vaccine. 2009;27(23):3045-52.

79. Murphy BR, Walsh EE. Formalin-inactivated respiratory syncytial virus vaccine induces antibodies to the fusion glycoprotein that are deficient in fusion-inhibiting activity. J Clin Microbiol. 1988;26(8):1595-7.

80. Polack FP, Hoffman SJ, Crujeiras G, Griffin DE. A role for nonprotective complement-fixing antibodies with low avidity for measles virus in atypical measles. Nat Med. 2003;9(9):1209-13.

81. de Swart RL, van den Hoogen BG, Kuiken T, Herfst S, van Amerongen G, Yuksel S, et al. Immunization of macaques with formalin-inactivated human metapneumovirus induces hypersensitivity to hMPV infection. Vaccine. 2007;25(51):8518-28.

82. Tregoning JS, Yamaguchi Y, Harker J, Wang B, Openshaw PJ. The role of $\mathrm{T}$ cells in the enhancement of respiratory syncytial virus infection severity during adult reinfection of neonatally sensitized mice. J Virol. 2008;82(8):4115-24.

83. Tseng CT, Sbrana E, Iwata-Yoshikawa N, Newman PC, Garron $\mathrm{T}$, Atmar RL, et al. Immunization with SARS coronavirus vaccines leads to pulmonary immunopathology on challenge with the SARS virus. PLoS ONE. 2012;7(4):e35421.

84. Bolles M, Donaldson E, Baric R. SARS-CoV and emergent coronaviruses: viral determinants of interspecies transmission. Curr Opin Virol. 2011;1(6):624-34. 
85. Yasui F, Kai C, Kitabatake M, Inoue S, Yoneda M, Yokochi S, et al. Prior immunization with severe acute respiratory syndrome (SARS)-associated coronavirus (SARS-CoV) nucleocapsid protein causes severe pneumonia in mice infected with SARS-CoV. J Immunol. 2008;181(9):6337-48.

86. Weingartl H, Czub M, Czub S, Neufeld J, Marszal P, Gren J, et al. Immunization with modified vaccinia virus Ankara-based recombinant vaccine against severe acute respiratory syndrome is associated with enhanced hepatitis in ferrets. J Virol. 2004;78(22):12672-6.

87. Wang Q, Zhang L, Kuwahara K, Li L, Liu Z, Li T, et al. Immunodominant SARS coronavirus epitopes in humans elicited both enhancing and neutralizing effects on infection in non-human primates. ACS Infect Dis. 2016;2(5):361-76.

88. Horstmann DM, Liebhaber H, Le Bouvier GL, Rosenberg DA, Halstead SB. Rubella: reinfection of vaccinated and naturally immune persons exposed in an epidemic. N Engl J Med. 1970;283(15):771-8.

89. Vennema H, DeGroot RJ, Harbour DA, Dalderup M, GruffyddJones T, Horzinek MC, Spaan WJM. Early death after feline infectious peritonitis challenge due to recombinant vaccinia virus immunization. J Virol. 1990;64:1407-9.

90. Vennema H, Poland A, Foley J, Pedersen NC. Feline infectious peritonitis viruses arise by mutation from endemic feline enteric coronaviruses. Virology. 1998;243(1):150-7.

91. Porter DDL, AE, Porter HG. Aleutian disease of mink. Adv Immunol. 1980;29:261-86.

92. Zhu HW, Xing XM, Wen YJ. Eenie, Meenie, Miney, Moe, who is responsible for the antibody-dependent enhancement of Aleutian mink disease parvovirus infection?. Chin J Virol. 2014;30(4):450-5.

93. Porter DD, Larsen AE, Porter HG. The pathogenesis of Aleutian disease of mink. I. In vivo viral replication and the host antibody response to viral antigen. J Exp Med. 1969;130:575-93.

94. Porter AD, Larsen AE, Porter HG. The pathogenesis of Aleutian disease of mink. II. Enhancement of tissue lesions following the administration of a killed virus vaccine or passive antibody. $\mathrm{J}$ Immunol. 1972;109:1-7.

95. Kanno H, Wohlinbarger JB, Bloom ME. Aleutian mink disease parvovirus infection of mink macrophages and human macrophage cell line *937: demonstration of antibody-dependent enhancement of infection. J Virol. 1993;67:7017-24.

96. Plagemann PG. Porcine reproductive and respiratory syndrome virus: origin hypothesis. Emerg Infect Dis. 2003;9(8):903-8.

97. Gómez-Laguna J, Salguero FJ, Pallarés FJ, Carrasco L. Immunopathogenesis of porcine reproductive and respiratory syndrome in the respiratory tract of pigs. Vet J. 2013;195(2):148-55.

98. Yoon KJ, Wu LL, Zimmerman JJ, Hill HT, Platt KB. Antibodydependent enhancement (ADE) of porcine reproductive and respiratory syndrome virus (PRRSV) infection in pigs. Viral Immunol. 1996;9(1):51-63.

99. Wan B, Chen X, Li Y, Pang M, Chen H, Nie X, et al. Porcine Fc $\gamma$ RIIb mediated PRRSV ADE infection through inhibiting IFN- $\beta$ by cytoplasmic inhibitory signal transduction. Int J Biol Macromol. 2019;1(138):198-206.

100. Iseki H, Kawashima K, Takagi M, Shibahara T, Mase M. Studies on heterologous protection between Japanese type 1 and type 2 porcine reproductive and respiratory syndrome virus isolates. $\mathbf{J}$ Vet Med Sci. 2020;82(7):935-42.

101. Zhou L, Ge X, Yang H. Porcine reproductive and respiratory syndrome modified live virus vaccine: a "Leaky" vaccine with debatable efficacy and safety. Vaccines. 2021;9(4):362. https:// doi.org/10.3390/vaccines9040362.

102. Kim JK, Seiler P, Forrest HL, Khalenkov AM, Franks J, Kumar $\mathrm{M}$, et al. Pathogenicity and vaccine efficacy of different clades of Asian $\mathrm{H} 5 \mathrm{~N} 1$ avian influenza A viruses in domestic ducks. J Virol. 2008;82(22):11374-82.

103. Mancera Gracia JC, Pearce DS, Masic A, Balasch M. Influenza A virus in swine: epidemiology, challenges and vaccination strategies. Front Vet Sci. 2020;7:647.

104. Chan CYY, Low JZH, Gan ES, Ong EZ, Zhang SL, Tan HC, et al. Antibody-dependent dengue virus entry modulates cell intrinsic responses for enhanced infection. mSphere. 2019;4(5):e.0052819. https://doi.org/10.1128/mSphere.00528-19.

105. Webster RG, Askonas BA. Cross-protection and cross reactive cytotoxic $\mathrm{T}$ cells induced by influenza virus vaccines in mice. Eur J Immunol. 1980;10:396-401.

106. Chanock RM, Parrott RH, Kapakian AZ. Possible role of immunological factors in pathogenesis of RS virus in lower respiratory tract disease. Perspect Virol. 1968;6:125-35.

107. King AA, Sands JJ, Porterfield JS. Antibody-mediated enhancement of rabies virus infection in a mouse macrophage cell line (P388D1). J Gen Virol. 1984;65:1091-3.

108. Prabhakar BS, Nathanson N. Acute rabies deaths mediated by antibody. Nature. 1981;290:590-5991.

109. Tirado SM, Yoon KJ. Antibody-dependent enhancement of virus infection and disease. Viral Immunol. 2003;16(1):69-86.

110. Burke DS. Human HIV vaccine trials: does antibody-dependent enhancement pose a genuine risk? Perspect Biol Med. 1992;35:511-30.

111. Mdurvwa EG, Ogunbiyi PO, Gakou HS, Reddy PG. Pathogenic mechanisms of caprine arthritis-encephalitis virus. Vet Res Commun. 1994;18(6):483-90.

112. Mealey RH, Leib SR, Littke MH, Wagner B, Horohov DW, McGuire TC. Viral load and clinical disease enhancement associated with a lentivirus cytotoxic $\mathrm{T}$ lymphocyte vaccine regimen. Vaccine. 2009;27(18):2453-68. 